DOI: https://doi.org/10.46296/ig.v4i8edespoct.0030

\title{
LOCALIZACIÓN DE PARES TRIBOLÓGICO EN BOMBAS DE AGUA TIPO CENTRIFUGAS PARA LA PROPUESTA DE UN PLAN DE MANTENIMIENTO INTEGRAL EN LA EMPRESA ATUNERA FISCHCORP S.A - MANTA
}

\section{LOCATION OF TRIBOLOGICAL PAIRS IN CENTRIFUGAL TYPE WATER PUMPS FOR THE PROPOSAL OF A COMPREHENSIVE MAINTENANCE PLAN IN THE COMPANY ATUNERA FISCHCORP S.A - MANTA}

\author{
Quiroz-Cedeño Danny Omar ${ }^{1}$; Sabando-Piguabe Luis Felipe ${ }^{2}$ \\ ${ }^{1}$ Maestrante de la Maestría de Investigación en Mantenimiento Industrial, Mención \\ Gestión Eficiente del Mantenimiento, Instituto de Posgrado, Universidad Técnica de \\ Manabí, UTM. Portoviejo, Ecuador. Correo: danny.quiroz1443@gmail.com. \\ 2 Universidad Técnica de Manabí, UTM. Portoviejo, Ecuador. Correo: \\ luis.sabandol@utm.edu.ec. ORCID ID: https://orcid.org/0000-0001-7492-7472
}

\begin{abstract}
Resumen
Los elementos mecánicos con pares tribológicos tales como las bombas de agua de uso industrial tipo centrífuga son equipos de primera línea de trabajo en las industrias de consumo alimenticios, en la actualidad la población ha crecido drásticamente por tal razón las industrias tratan de optimizar las operatividades de estos equipos que contienen pares tribológicos debido a las series de desgastes que estos presentan al momento de estar en función y más aún si carecen de un mantenimiento adecuado y periódico. Mantenimiento es un término muy empleado en el día a día de las personas y de forma genérica y amplia. Parece una tarea difícil definir el mantenimiento desde el punto de vista industrial. La mayoría de las personas entiende por mantenimiento el acto de arreglar y reparar equipos e instalaciones para asegurar la continuidad operativa, es decir, conservación y no mantenimiento, pero, además, es mantener, prevenir, analizar, mejorar continuamente, dominar tecnológicamente y económicamente los medios productivos con seguridad y preservación del medio ambiente, el mantenimiento debe iniciarse bajo un control estricto de medidas técnicas y direccionadas en la condición de cada activo, llevando acabo una definición de la política de mantenimiento, organización de la documentación, registro y suministro de repuestos y procedimientos adecuados. En este proyecto se diagnosticarán los fallos funcionales en las bombas de agua tipo centrífugas y equipos de bombeos en general, estos son los excesivos rozamientos que se presentan en las zonas habituales tales como: ejes, rodamientos, sellos, etc.
\end{abstract}

Palabras clave: Fricción, desgaste, lubricación, Tribología, Cavitación, Estectocoipio, viscosímetro, termómetro.

\footnotetext{
Abstract

The mechanical elements with tribological pairs such as centrifugal type water pumps for industrial use are the first line of work equipment in the food consumption industries, nowadays the population has grown drastically for this reason the industries try to optimize the operation of these equipments that contain tribological pairs due to the series of wear that they present at the moment of being in function and even more if they lack an adequate and periodic maintenance. Maintenance is a term widely used in the daily life of people and in a generic and broad way. It seems a difficult task to define maintenance from an industrial point of view. Most people Información del manuscrito:
}

Fecha de recepción: 02 de agosto de 2021.

Fecha de aceptación: 01 de octubre de 2021.

Fecha de publicación: 05 de octubre de 2021. 
understand maintenance as the act of fixing and repairing equipment and facilities to ensure operational continuity, i.e. conservation and not maintenance, but, in addition, it is to maintain, prevent, analyze, continuously improve, technologically and economically master the productive means with safety and preservation of the environment. Maintenance must be initiated under a strict control of technical measures and directed to the condition of each asset, carrying out a definition of the maintenance policy, organization of documentation, registration and supply of spare parts and adequate procedures. This project will diagnose the functional failures in centrifugal type water pumps and pumping equipment in general, these are the excessive frictions that occur in the usual areas such as: shafts, bearings, seals, etc.

Keywords: Friction, wear, lubrication, Tribology, Cavitation, Stectocoipium, viscometer, thermometer.

\section{Introducción}

\section{Antecedentes Investigativos}

La primera bomba construida por el hombre fue la jeringa y se debe a los antiguos egipcios, quienes la utilizaron para embalsamar las momias. En el siglo II A.C., esta idea o principio ocurrido en la jeringa se convirtió en una fuerza hidráulica de empuje de doble efecto. Con el pasar del tiempo estos sistemas fueron perfeccionando en base a los estudios realizados en pequeños ensayos prácticos, en la actualidad el estudio que se realiza sobre los parámetros de bombas centrífugas no está basado solo en lo teórico, muchas instituciones educativas de nivel superior tienen la factibilidad de realizar dichos estudios de una forma aplicada para obtener $y$ comprobar las características fundamentales de las bombas.
Se establece que en base a estudios previos antes de una selección es importante conocer a qué condiciones la bomba va a estar funcionando, esto se lo realiza para que el ingeniero encargado en seleccionar la bomba este totalmente seguro en que esta abastezca la necesidad deseada de empuje de agua.

En nuestro país universidades como la Politécnica Nacional del Ecuador, Universidad Tecnológica Equinoccial y la Politécnica de Chimborazo, realizan de forma minuciosa el estudio de parámetros característicos de bombas centrífugas, aportando a la formación experimental de sus estudiantes y por ende ser competitivos en el área de campo. (Ramírez Pozo, 2012) 


\section{Objetivo de la investigación}

La finalidad de este proyecto es recopilar la información necesaria de los datos históricos de fallos funcionales entre otros, estos procedimientos se ejecutan con ideas claras para proponer al personal del área de bombeo de agua potable un plan de mantenimiento integral tales como preventivos, proactivos y correctivos, mediante la modificación de las ordenes de trabajo para cada activo de acuerdo a la necesidad que este lo requiera y que esta propuesta sea aceptable y que apliquen dicha ejecución notándose las mejoras continuas, minimizando los fallos funcionales en el área de lavado, autoclaves, calderos, en la empresa fischcorp SA de la ciudad de manta.
A continuación, denotaremos algunos de los procedimientos más relevantes de la investigación:

Recopilar información histórica de operatividad de cada uno de los equipos

> Detección de anomalías físicas, durante el funcionamiento de los equipos utilizando instrumentos tales como estetoscopios, termómetros, densímetros etc.

> Llevar un control de calidad en los componentes mecánicos a reemplazar

$>$ Elaborar un plan de mantenimiento preventivo $\mathrm{y}$ correctivo en los equipos de bombeo.

Recopilar información práctica para la aplicación de los mantenimientos adecuados.

Tabla 1. Daños localizados en la Empresa Atunera Fischcorp S.A. - Manta.

\begin{tabular}{|c|c|c|c|c|}
\hline No. & Causa registrada & $\begin{array}{c}\text { Cantidad de } \\
\text { equipos }\end{array}$ & Porcentaje \% & \% Acumulado \\
\hline 1 & $\begin{array}{c}\text { Fuga de líquidos por } \\
\text { sellos mecánicos }\end{array}$ & 36 & 63,16 & 63,16 \\
\hline 2 & $\begin{array}{c}\text { Daños en elementos que } \\
\text { conforman la junta entre } \\
\text { casco y cabezotes }\end{array}$ & 5 & 8,77 & 71,93 \\
\hline 3 & $\begin{array}{c}\text { Formación de fluoruro en } \\
\text { partes internas de las } \\
\text { bombas }\end{array}$ & 4 & 7,02 & 78,95 \\
\hline 4 & Desbalances & 4 & 7,01 & 85,96 \\
\hline
\end{tabular}




\begin{tabular}{|c|c|c|c|c|}
\hline 5 & $\begin{array}{c}\text { Partes desprendidas } \\
\text { dentro de la bomba } \\
\text { (impulsor y aro) }\end{array}$ & 3 & 5,27 & 91,23 \\
\hline 6 & Desalineación & 3 & 5,26 & 96,49 \\
\hline 7 & Rodamientos dañados & 1 & 1,76 & 98,25 \\
\hline 8 & Holgura excesiva & 1 & 1,75 & 100,00 \\
\hline
\end{tabular}

Ilustración 1. Diagrama de Pareto obtenido con los fallos registrados en la Empresa Atunera Fischcorp S.A. - Manta.

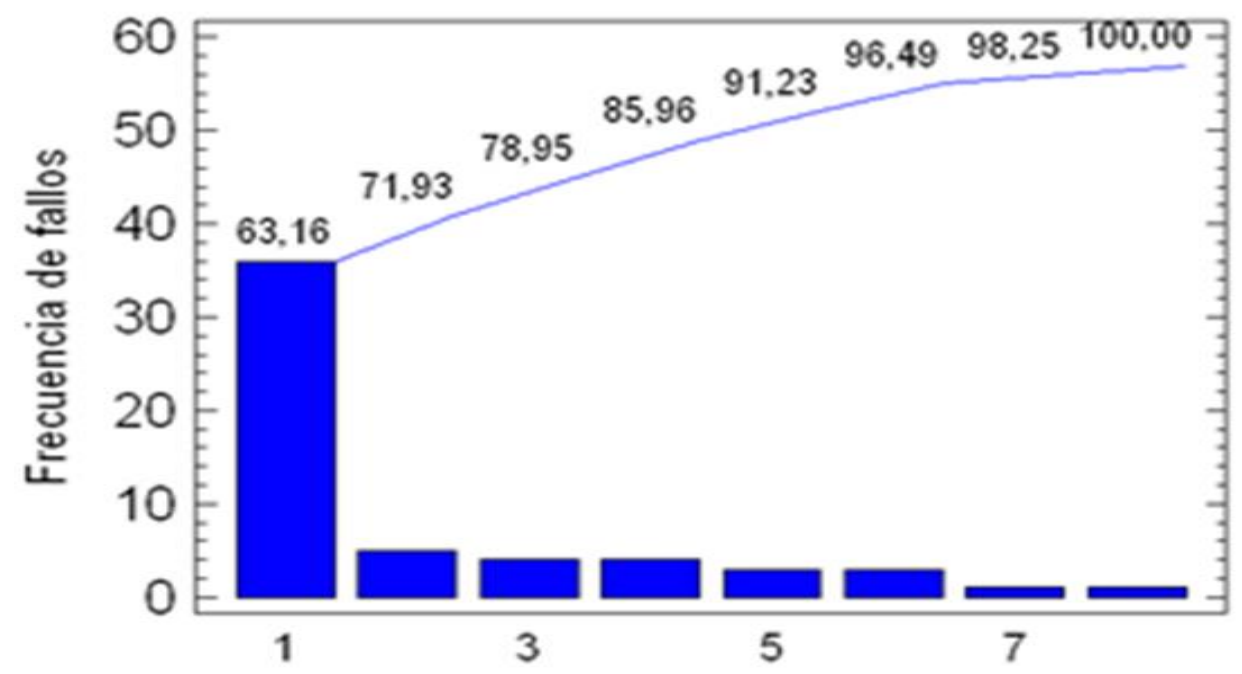

\section{Metodología}

\section{Método de estudio a utilizar en el proyecto}

El método de estudio investigativo es técnico - analítico, Ya que se toman conclusiones generales para explicarlas de manera particular a los operadores, tomándose como referencia la ejecución de un análisis minucioso de las respectivas recopilaciones documentos puntualizados de acuerdo a cada necesidad requerida por cada activo.
Se refiere a los datos e historiales suscitados durante las operaciones efectuadas en cada equipo de bombeo que esté en función, bajo este régimen se harán comparaciones con otras fichas técnicas, para determinar acciones proactivas necesarias correspondientes en cada uno de los activos, para las mejoras de confiabilidad. 
Tabla 2. Programa general de la investigación.

\begin{tabular}{|c|c|c|c|c|c|}
\hline \multicolumn{6}{|c|}{ PROGRAMA GENERAL DE LA INVESTIGACIÓN } \\
\hline AREA & OBJETO DE ESTUDIO & ASPECTOS ANALIZADOS & INSTRUMENTOS & LUGAR & LOCALIDAD \\
\hline $\begin{array}{l}\text { Planta de bombeo de agua } \\
\text { potable }\end{array}$ & \begin{tabular}{|l|} 
Análisis de \\
instalación y \\
estructuras de las \\
bomba tipo \\
centrifuga y equipos \\
de bombeo.
\end{tabular} & $\begin{array}{l}\text { Reportes de horas de } \\
\text { trabajos; } \\
\text { Ordenes de Trabajo para } \\
\text { los diagnósticos previos a } \\
\text { la ejecución de los } \\
\text { mantenimientos Correctivo } \\
\text { y Preventivo). }\end{array}$ & \begin{tabular}{|l} 
Tecnicas de \\
diagnósticos a \\
través de la \\
inspección \\
visual. \\
Herramientas \\
mecánicas \\
manuales.
\end{tabular} & $\begin{array}{l}\text { Empresa Atunera } \\
\text { FISCORP SA. }\end{array}$ & $\begin{array}{l}\text { Planta de bombeo } \\
\text { de agua potable de } \\
\text { la Empresa Atunera } \\
\text { FISCORP SA. }\end{array}$ \\
\hline $\begin{array}{c}\text { Bombas de agua tipo centrifuga, } \\
\text { Equipos de bombeos. }\end{array}$ & $\begin{array}{l}\text { Fichas tecnicas para } \\
\text { la recopilación de } \\
\text { datos e históricos de } \\
\text { fallos }\end{array}$ & $\begin{array}{l}\text { Órdenes para la aplicación } \\
\text { de Mantenimiento } \\
\text { respectivos } \\
\text { Fichas técnicas para el } \\
\text { ingreso de los datos } \\
\text { de costo }\end{array}$ & $\begin{array}{l}\text { Suministros de } \\
\text { Oficina, } \\
\text { bitácoras: fichas } \\
\text { tecnicas, check } \\
\text { list, } \\
\text { Esferográficos } \\
\text { Calculadoras. } \\
\text { laptop Etc. }\end{array}$ & $\begin{array}{l}\text { Departamento de } \\
\text { mantenimiento del } \\
\text { Área de bombeo de } \\
\text { la Empresa Atunera } \\
\text { FISCORP SA. de la } \\
\text { ciudad de Manta. }\end{array}$ & $\begin{array}{l}\text { Área de bombeos } \\
\text { de agua potable, } \\
\text { Equipos de } \\
\text { bombeos de la } \\
\text { Empresa Atunera } \\
\text { FISCORP SA. De la } \\
\text { ciudad de Manta. }\end{array}$ \\
\hline $\begin{array}{l}\text { Zonas afectadas por la presencia } \\
\text { de pares tribológicos por un } \\
\text { inadecuado mantenimiento. }\end{array}$ & \begin{tabular}{|l|} 
Localización de pares \\
tribológicos en \\
bombas centrifugas y \\
equipos de bombeo \\
de la Empresa \\
Atunera FISCORP SA. \\
de la ciudad de \\
Manta
\end{tabular} & $\begin{array}{l}\text { Ordenes de trabajo } \\
\text { para la ejecución del plan } \\
\text { de mantenimiento integral } \\
\text { en bombas de agua tipos } \\
\text { centrifugas. }\end{array}$ & $\begin{array}{l}\text { Densímetros } \\
\text { Estetoscopios } \\
\text { Termógrafos, } \\
\text { Caudalímetros. }\end{array}$ & $\begin{array}{l}\text { Departamento de } \\
\text { mantenimiento del } \\
\text { Área de bombeo de } \\
\text { la Empresa Atunera } \\
\text { FISCORP SA. de la } \\
\text { ciudad de Manta. }\end{array}$ & $\begin{array}{l}\text { Área de bombeos } \\
\text { de agua potable de } \\
\text { la Empresa Atunera } \\
\text { FISCORP SA. De la } \\
\text { ciudad de Manta. }\end{array}$ \\
\hline
\end{tabular}

Tabla 3. Definición de las variables.

\begin{tabular}{|c|c|c|}
\hline \multicolumn{3}{|c|}{ DEFINICIÓN DE LAS VARIABLES } \\
\hline VARIABLES & TIPOS DE VARIABLES & CONCEPTOS \\
\hline $\begin{array}{l}\text { LOCALIZACIÓN DE PARES } \\
\text { TRIBOLOGICOS EN BOMBAS DE } \\
\text { AGUA TIPO CENTRÍFUGAS }\end{array}$ & DEPENDIENTE & $\begin{array}{l}\text { Localizar los pares } \\
\text { tribológicos existentes en } \\
\text { las bombas de agua tipo } \\
\text { centrifuga y grupos de } \\
\text { bombeos en general. }\end{array}$ \\
\hline $\begin{array}{c}\text { PLÁN DE MANTENIMIENTO } \\
\text { INTEGRAL }\end{array}$ & INDEPENDIENTE & $\begin{array}{l}\text { Llevar un control através de } \\
\text { fichas tecnicas para separar } \\
\text { las bombas tipos } \\
\text { centrifugas con los grupos } \\
\text { de bombeo, para } \\
\text { determinar la aplicación de } \\
\text { mantenimiento a cada uno } \\
\text { de los activos. }\end{array}$ \\
\hline
\end{tabular}


Tabla 4. Operacionalización de las variables.

\begin{tabular}{|c|c|c|c|c|c|c|}
\hline \multicolumn{7}{|c|}{ OPERACIONALIZACIÓN DE LA VARIABLE } \\
\hline $\begin{array}{c}\text { PARES } \\
\text { TRIBOLOGICOS }\end{array}$ & $\begin{array}{l}\text { Fallos funcionales } \\
\text { por deficiencia en } \\
\text { la gestión del } \\
\text { mantenimiento. }\end{array}$ & $\begin{array}{l}\text { Variable dependiente: } \\
\text { Por su naturaleza es } \\
\text { cuantitativa, por su grado } \\
\text { de complejidad es: } \\
\text { complicado. }\end{array}$ & $\begin{array}{l}\text { Según Calderón Malla \& Montalván } \\
\text { Pulla afirma que las diferentes causa- } \\
\text { raíz que llevan al fallo de rodamiento se } \\
\text { dan por excesivo trabajo sometidos a } \\
\text { altas temperatura. Generalmente, hay } \\
\text { tres tipos de fallo por fatiga: picadura } \\
\text { por fatiga pitting, desprendimiento por } \\
\text { fatiga spalling y desgaste de las } \\
\text { superficies. }(2019, \text { p. } 60)\end{array}$ & $\begin{array}{l}\text { Diagnóstico de } \\
\text { precisión sobre el } \\
\text { tiempo de vida útil } \\
\text { de una bomba } \\
\text { centrifuga, según su } \\
\text { uso. }\end{array}$ & $\begin{array}{l}\text { Variedades de } \\
\text { modelos de los } \\
\text { equipos de bombeo }\end{array}$ & $\begin{array}{l}\text { Guías de } \\
\text { observación }\end{array}$ \\
\hline $\begin{array}{l}\text { EN BOMBAS DE } \\
\text { AGUA TIPO } \\
\text { CENTRIFUGAS, } \\
\text { PARA USO } \\
\text { INDUSTRIAL }\end{array}$ & $\begin{array}{l}\text { Aplicacion de } \\
\text { mantenimiento } \\
\text { integral a los } \\
\text { equipos de } \\
\text { bombeo de } \\
\text { interés }\end{array}$ & $\begin{array}{l}\text { Variable independiente: } \\
\text { Por su naturaleza es } \\
\text { cualitativa, por su grado } \\
\text { de complejidad es: } \\
\text { complicado. }\end{array}$ & $\begin{array}{l}\text { De acuerdo a Cruz (2013, p. 13) se } \\
\text { determina que tradicionalmente, se } \\
\text { han distinguido } 4 \text { tipos de } \\
\text { mantenimiento, que se diferencian } \\
\text { entre sí, por el carácter de las tareas } \\
\text { que se incluyen según el requerimiento } \\
\text { o expectativas: } \\
\text { Mantenimiento correctivo } \\
\text { Mantenimiento preventivo } \\
\text { Mantenimiento predictivo } \\
\text { Mantenimiento Proactivo. }\end{array}$ & $\begin{array}{l}\text { Tipo de } \\
\text { mantenimiento } \\
\text { aplicado durante la } \\
\text { operación del } \\
\text { equipo. }\end{array}$ & $\begin{array}{c}\text { Matriz de registros } \\
\text { del historial de los } \\
\text { equipos. }\end{array}$ & $\begin{array}{l}\text { Guías de } \\
\text { observación }\end{array}$ \\
\hline
\end{tabular}

\section{Resultados y discusión}

\section{Resultados}

Mediante la ejecución del trabajo realizado en base a la localización de pares tribológico en bombas de agua tipo centrifugas para la propuesta de un plan de mantenimiento integral en la empresa atunera Fischcorp S.A manta, se obtuvieron resultados positivos en cuanto a mejoras continuas, estos análisis y asistencias técnicas analíticas involucran principalmente a los sistemas de lubricación y desgastes prematuros en los elementos móviles de los equipos de bombeos en general, de dicha empresa de enlatados de consumos masivos.

En la investigación realizada por las diferentes sesiones realizadas se pudo recopilar mucha información, estas van desde la toma de datos históricos de fallos hasta el itinerario de mantenimiento que se le dieron a cada equipo de bombeo durante su funcionamiento.

Puedo resumir que en condiciones ideales las bombas pueden funcionar casi indefinidamente y que en condiciones adversas se descomponen a menudo, sin embargo, se tomó muy en cuenta la 
característica del fluido que se bombea, ya sea este corrosivo 0 abrasivo.

Cabe recalcar que con los mantenimientos apropiados en estas condiciones de trabajo la vida de cualquier bomba se puede extender. Por otro lado se determinó que la mayoría de daños prematuros de una bomba son causados por la contaminación, por la lubricación incorrecta 0 por problemas de alineación.

Para retrasar las fallas se deben realizar procedimientos correctos en la operación de arranque de la bomba estos son el cebado, las verificaciones de rutina y el cierre (paro).

Es muy importante cerciorarse de que la bomba esté totalmente llena de líquido antes de arrancarla.

Se utilizaron instrumentos de mediciones tales como termómetros, estetoscopios, densímetros para las detecciones de anomalías tales como: vibraciones, altas temperaturas y calidad de los aceites lubricantes.

Otro problema que se pudo determinar fue la causa principal que producen los daños en los empaques, generalmente estos son los problemas de lubricación que hacen que los empaques se carbonicen y presenten un aspecto vidriado, conllevando al sobrecalentamiento de la bomba.

Cabe recalcar que para llevar un control programado en el cambio de aceite $y$ al mismo el tiempo monitorear los tiempos de vida de cada uno de los elementos mecánicos y poderlos reemplazar en los rangos de fechas dados por el fabricante, se elaboraron hojas de trabajos tales como fichas técnicas, Check list, bitácoras diarias etc.

Este proceso técnico se lo aplicó para llevar un control óptimo en el funcionamiento mediante los mantenimientos programados a cada uno de los activos según la necesidad que estos requerían. 


\section{Discusión}

El Informe de Confiabilidad (IC) tiene la función de calcular un índice de confiabilidad para un servicio de mantenimiento ejecutado en una bomba centrífuga y que através de la funcionalidad del equipo en tratamiento podemos destacar la cuantificación de la calidad del servicio realizado, representado por un promedio final.

El número de re-trabajo fue elegido como indicador de la calidad del servicio, consecuentemente con el índice de confiabilidad. Por lo tanto, el Índice de Retraso (IR) está íntimamente relacionado con la calidad del servicio. $Y$ de esta manera cuantificar la calidad del trabajo o cuidado, en como las tareas de servicio de mantenimiento son realizados y la capacitación de quien las ejecuta, esto significa actuar directamente en el re-trabajo.

Las herramientas de trabajo, el Informe de Confiabilidad (IC) y la Matriz de Habilidades (MH), involucran la consideración de los tres aspectos de los factores humanos: características de la tarea, características personales y aspecto organizativo.
El análisis de estos tres aspectos y de su impacto sobre la calidad de los servicios a través de la aplicación de la Matriz de Habilidades (MH) generaron resultados positivos en la reducción del indicador Índice de Retraso (IR).

Mientras que la Matriz de Habilidades $(\mathrm{MH})$ implica sólo las entrevistas actualizadas periódicamente, el Informe de Confiabilidad (IC) evalúa estas competencias y genera una base de datos a cada servicio realizado. 
Tabla 5. Fallos funcionales de bombas de agua tipo centrifuga.

\begin{tabular}{|c|c|c|}
\hline SINTOMA & CAUSA & CORRECCIÓN \\
\hline \multirow{3}{*}{ La bomba no arranca } & $\begin{array}{l}\text { No funciona el motor eléctrico, no hay } \\
\text { energia eléctrica o el motor está quemado }\end{array}$ & Checar que exista energía eléctrica. Desmontar y embobinar \\
\hline & $\begin{array}{l}\text { Elementos térmicos "botados" o fusibles } \\
\text { quemados }\end{array}$ & Restablecerlos y verificar el buen funcionamiento del motor \\
\hline & Falsos contactos & Revisar toda la instalación y reapretar \\
\hline \multirow{6}{*}{$\begin{array}{l}\text { La bomba funciona, } \\
\text { pero no hay entrega } \\
\text { de agua }\end{array}$} & La bomba tiene aire & $\begin{array}{l}\text { Purgar la bomba, llenando completamente de agua el tubo } \\
\text { de succión y la caja de agua }\end{array}$ \\
\hline & Velocidad demasiado baja & Revisar el voltaje del motor y la frecuencia \\
\hline & Altura de descarga demasiado grande & $\begin{array}{l}\text { Verificar que las válvulas a la salida estén totalmente abiertas, } \\
\text { analizar pérdidas de fricción y corregir el problema }\end{array}$ \\
\hline & Altura de succión demasiado grande & $\begin{array}{c}\text { Si no hay obturación en la admisión, la bomba no succiona bien debido } \\
\text { a una mala elección del equipo. Probablemente bajando el nivel } \\
\text { de la bomba se solucione }\end{array}$ \\
\hline & Impulsor parcial o totalmente bloqueado & Destapar la caja de la bomba y limpiar bien el impulsor \\
\hline & Incorrecta dirección de la rotación & Verificar la rotación del motor y corregir intercambiando fases \\
\hline \multirow{8}{*}{$\begin{array}{l}\text { Insuficiente entrega } \\
\text { de agua }\end{array}$} & Aire en el tubo de succión & Revisar estado fisico del tubo y tapar cualquier orificio que se encuentre \\
\hline & Aire en la prensa estopa & $\begin{array}{l}\text { Si no hay un leve chorro delgado de agua en la prensa estopa, un ajuste } \\
\text { no forzado deja de producirlo, conviene cambiar empaquetadura; } \\
\text { de no ser éste el caso, limpiar el tubo de agua de cierre }\end{array}$ \\
\hline & $\begin{array}{l}\text { El agua caliente está cercana al punto de } \\
\text { ebullición }\end{array}$ & $\begin{array}{l}\text { Se constata por la oscilación permanente del manómetro; } \\
\text { aumentar la altura del tanque de condensados }\end{array}$ \\
\hline & $\begin{array}{l}\text { Anillos de desgaste defectuosos } \\
\text { (los que protegen al impulsor) }\end{array}$ & Examinarlos y cambiarlos \\
\hline & Impulsor defectuoso & Revisarlo, renovarlo o cambiarlo \\
\hline & Empaquetadura defectuosa & Cambiar empaques y bujes si estản desgastados \\
\hline & La válvula de pie es más chica & $\begin{array}{l}\text { En el caso de usarse colador, el espacio útil total debe ser de tres } \\
\text { a cuatro veces mayor que el espacio del tubo de aspiración }\end{array}$ \\
\hline & $\begin{array}{l}\text { El nivel del agua está bajo y tambièn } \\
\text { succiona aire }\end{array}$ & $\begin{array}{l}\text { Si sucede comúnmente, bajar el tubo de succión; si es eventual, } \\
\text { esperar a que el nivel suba }\end{array}$ \\
\hline La presión disminuye & Aumenta el gasto de agua & La demanda es mayor que la existente \\
\hline \multirow{4}{*}{ Se calienta el motor } & Prensa estopa demasiado apretada & Aflojar la presión del casquillo; apretarlo sin exceso, si hay necesidad \\
\hline & Caja deformada por el peso de la tubería & $\begin{array}{c}\text { Examinar caja de la bomba para revisar que no exista roce } \\
\text { con el impulsor y que los anillos de desgaste están en buen estado. } \\
\text { Cambiar piezas desgastadas }\end{array}$ \\
\hline & Conjunto desalineado & Venificar alineación correcta del motor y de la bomba, colocar calzas necesarias \\
\hline & Flecha desbalanceada & $\begin{array}{l}\text { Si no es ninguna de las causas anteriores, puede ser la flecha o el cople. } \\
\text { Reparar o cambiar }\end{array}$ \\
\hline $\begin{array}{l}\text { Constantemente hay que } \\
\text { rectificar la flecha }\end{array}$ & Bujes desgastados & Cambiar los bujes y posiblemente cambiar empaque del prensa estopas \\
\hline
\end{tabular}

Obtenido de: Bombas Centrífugas - Revista Cero Grados (Ogrados.com.mx) 
Ilustración 2. Rendimiento de las bombas centrifugas Caudal (I/min) vs Rendimiento (\%)

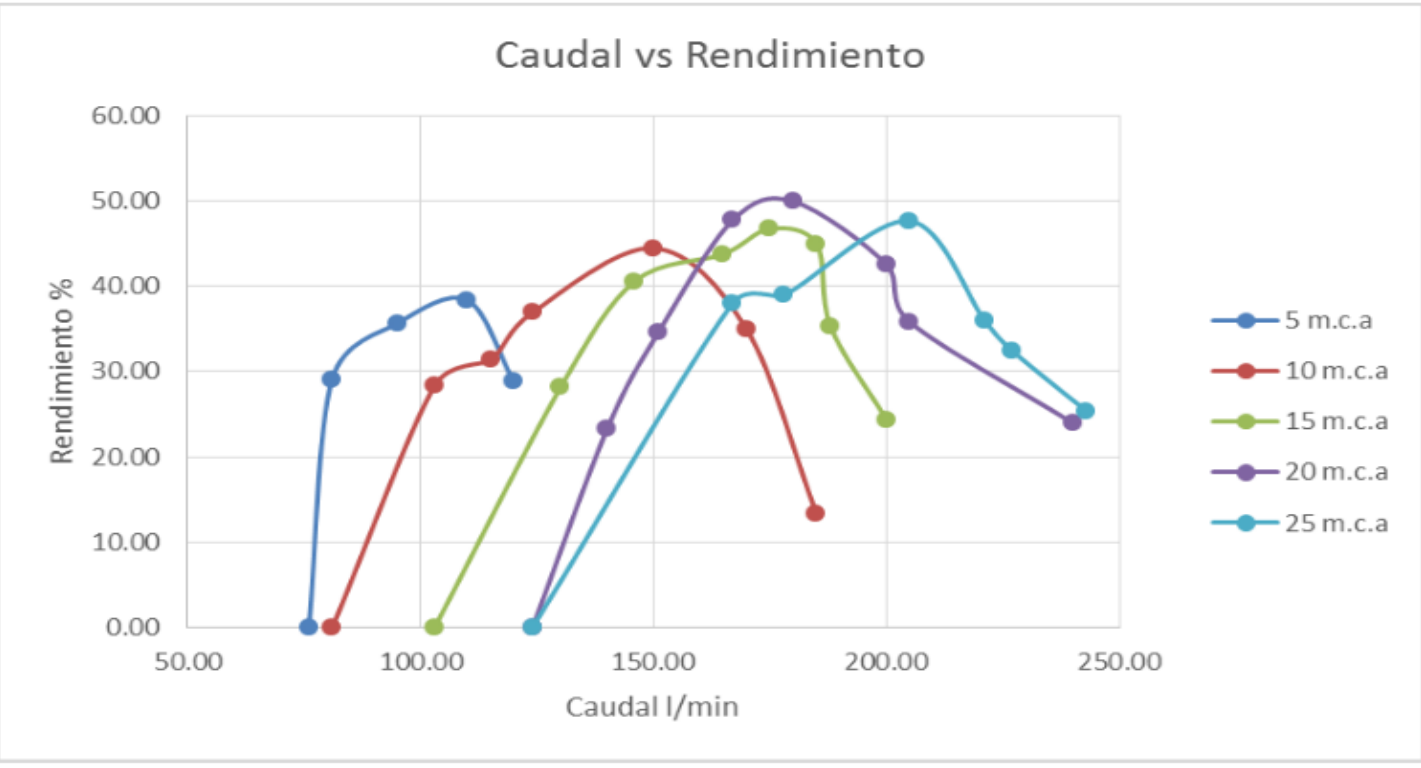

Obtenido de: (Garay Quintana 2016)

Ilustración 3. Rendimiento de las bombas centrifugas Caudal (m3/h) vs $H(m)$

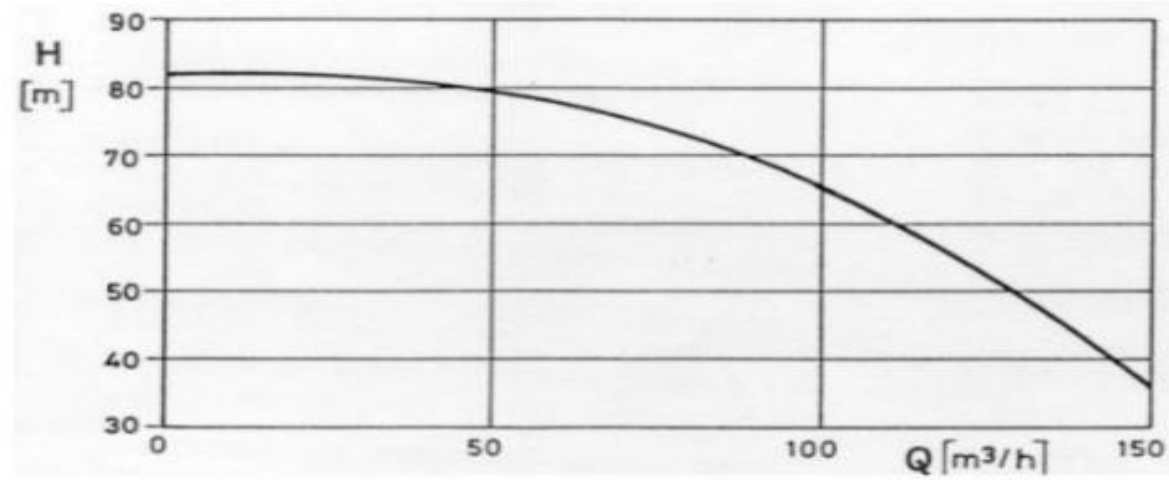

Obtenido de: Curva característica de la bomba centrífuga / Debem Srl

Ilustración 4. Rendimiento de las bombas centrifugas Caudal (m3/h) vs $\eta(\%)$

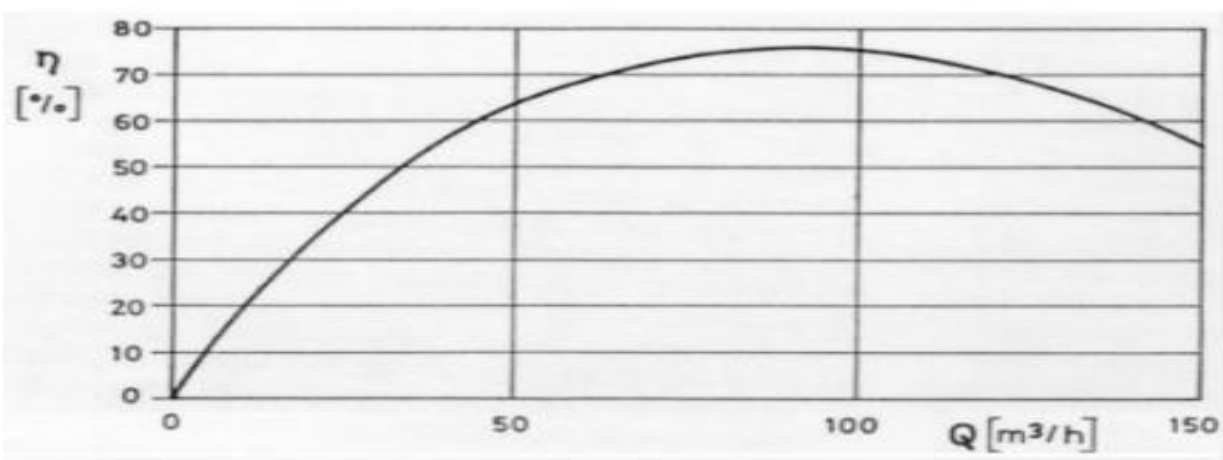

Obtenido de: Curva característica de la bomba centrífuga / Debem Srl 
Ilustración 5. Rendimiento de las bombas centrifugas Caudal (m3/h) vs W (cv)

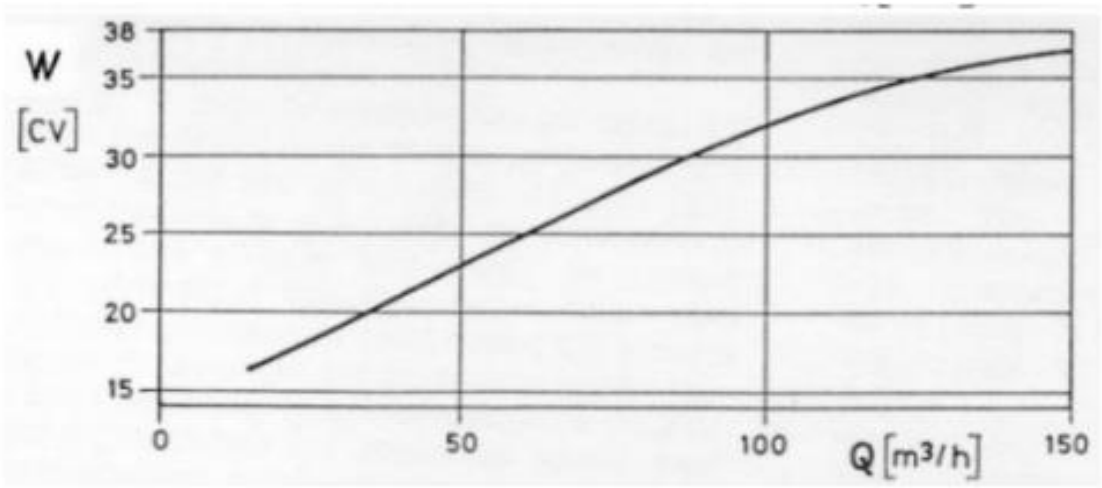

Obtenido de: Curva característica de la bomba centrífuga / Debem Srl

Tabla 6. Condiciones de balanceo de sellos de la bomba de agua tipo centrífuga.

\begin{tabular}{|l|l|l|}
\hline \multicolumn{1}{|c|}{ Descripción } & \multicolumn{1}{|c|}{ Sello balanceado } & \multicolumn{1}{|c|}{ Sello no balanceado } \\
\hline $\begin{array}{l}\text { Generación de calor en } \\
\text { las caras del sello }\end{array}$ & Menor & Mayor \\
\hline Vida útil & Mayor & Menor \\
\hline Carga en la cara & Baja & Alta \\
\hline $\begin{array}{l}\text { Presión en el } \\
\text { prensaestopas }\end{array}$ & $\begin{array}{l}\text { Indicado para bombas } \\
\text { de todos los rangos de } \\
\text { presión }\end{array}$ & $\begin{array}{l}\text { Indicado para bombas } \\
\text { con baja presión en el } \\
\text { prensaestopas }\end{array}$ \\
\hline $\begin{array}{l}\text { Vibración y } \\
\text { desalineación }\end{array}$ & Menos estable & Más estable \\
\hline $\begin{array}{l}\text { Fluidos recomendados } \\
\text { para el uso }\end{array}$ & $\begin{array}{l}\text { Líquidos y gases con } \\
\text { poca lubricidad y altas } \\
\text { presiones de vapor, } \\
\text { tales como } \\
\text { hidrocarburos ligeros }\end{array}$ & $\begin{array}{l}\text { Líquidos o gases con } \\
\text { muy buena lubricidad }\end{array}$ \\
\hline
\end{tabular}

Obtenido de: Cómo elegir sellos mecánicos / De Máquinas y Herramientas

\section{(demaquinasyherramientas.com)}

\section{Conclusiones}

En la investigación realizada por las diferentes sesiones realizadas se recopilaron mucha información, estas van desde la toma de datos históricos de fallos hasta el itinerario de mantenimiento que se le dieron a cada equipo durante su funcionamiento.
Puedo resumir que en condiciones ideales que las bombas pueden funcionar casi indefinidamente y que en condiciones adversas se descomponen a menudo, sin embargo se tomó muy en cuenta la característica del fluido que se bombea, ya sea este corrosivo o abrasivo. 
Cabe recalcar que con la aplicación de mantenimientos apropiados en estas condiciones de trabajo la vida de cualquier bomba de puede extender.

Por otro lado se determinó que la mayoría de daños prematuros de una bomba son causados por la contaminación, por la lubricación incorrecta o por problemas de alineación.

Para retrasar las fallas se deben realizar procedimientos correctos en la operación de arranque de la bomba estos son el cebado, las verificaciones de rutina y el cierre (paro)

Es muy importante cerciorarse de que la bomba esté totalmente llena de líquido antes de arrancarla.

Se utilizaron instrumentos de mediciones tales como termómetros, estetoscopios, densímetros para las detecciones de anomalías tales como: vibraciones, altas temperaturas y calidad de los aceites lubricantes.

El consumo excesivo de energía eléctrica puede ser el resultado de las presiones insuficientes cuyas causas fueron mencionadas con anterioridad y de las siguientes condiciones: desalineación, partes desgastadas o dañadas, problemas de cojinetes etc.

Otro problema que se pudo determinar fue la causa principal que producen los daños en los empaques, generalmente estos son los problemas de lubricación que hacen que los empaques se carbonicen provocando un recalentamiento en la bomba.

\section{Recomendaciones}

La recomendación principal es verificar que el plan de mantenimiento propuesto se esté realizando en su totalidad sin dejar a un lado la perseverancia de su aplicación durante el funcionamiento de cada equipo de bombeo.

A continuación, detallaremos algunas recomendaciones:

> Optimización de la disponibilidad del equipo productivo.

> Disminución de los costes por cada mantenimiento aplicado

$>$ Optimizar los recursos eficientemente.

- Maximizar la vida de la máquina, equipo, instalación, etc. 
$>$ Evitar en la mayor medida posible, o en su caso reducir, la aparición de fallos.

Evitar las paradas innecesarias de la bomba.

$>$ Evitar accidentes y aumentar la seguridad de las personas.

- Llevar un control de calidad en los componentes mecánicos a reemplazar

Recopilar información práctica para la aplicación de los mantenimientos adecuados

\section{Bibliografía}

Bonilla Villalba, A. G. and J. A. Núñez Villacrés (2020). Diseño y simulación de un impulsor centrífugo radial semi abierto para bombas de torbellino,

Ecuador:

Latacunga:

Universidad

Técnica de Cotopaxi;

Facultad de Ciencias de....

Calderón Malla, J. C., \& Montalván Pulla, F. I. (2019). Adquisición de señales acústicas y vibracionales para el diagnóstico de fallos en una bomba centrífuga multietapas de eje vertical

Carrasco, F. J. C. (2016). Características de los sistemas TPM y RCM en la ingeniería del mantenimiento.
Choque Apaza, E. S. (2019). "Implementación de un sistema de gestión del mantenimiento de bombas centrífugas a través del monitoreo subjetivo."

Cruz, A. M. E. (2013). ESTUDIO DE UN SISTEMA DE MANTENIMIENTO PREDICTIVO BASADO EN ANÁLISIS DE VIBRACIONES IMPLANTADO EN INSTALACIONES DE BOMBEO Y GENERACIÓN.

Cruzado Valladares, R. J. (2020). "Aplicación del mantenimiento centrado en la confiabilidad (RCM) a bombas de carga en una refinería."

Garay Quintana, E. J. (2016). "Configuración de los componentes de una bomba centrífuga utilizada como turbina para mejorar el rendimiento en una pico central hidroeléctrica."

Garrido Martínez, S. (2017). Diseño y estudio de una bomba hidráulica centrífuga mediante la generación de su prototipo virtual.

Grández Noriega, L. G. (2018). Sistema de bombeo de agua para mejorar el proceso de succión y riego de los vehículos cisternas de la empresa Construcción y Administración SA-Bagua 2018. 
Hermosilla Pérez, A. M. (2017). Emisiones acústicas como precursor de daño para caracterizar la degradación en una bomba centrífuga.

Hernández, M. (2018). Evaluación de la competitividad de la industria atunera en la economía de la ciudad de Manta-Ecuador período 20122016 Universidad de Guayaquil. Facultad de Ciencias Económicas].

Lucas, F. S. (2017). LA CALIDAD Y COMPETITIVIDAD EN LAS EMPRESAS DE LA CIUDAD DE MANTA-ECUADOR. Mikarimin. Revista Científica Multidisciplinaria. e-ISSN 2528-7842, 3(3), 09-18.

MUÑOZ JARA, F. G. and M. A. GUTIÉRREZ ZAPATA (2018). "MEJORAMIENTO BANCO DE PRUEBAS PARA BOMBA CENTRÍFUGA."

Noriega Córdova, M. H., Simbaña Guallimba, D. J., \& Torres Díaz, D. E. (2018). Estudio tribológico en función de la temperatura utilizando aditivos no sólidos.

Ramírez Pozo, E. A. (2012). Estudio de bombas centrífugas y su factibilidad de aplicación en la Facultad de Ingeniería Mecánica con la finalidad de obtener parámetros técnicos con variación de caudal Universidad Técnica de Ambato. Facultad de
Ingeniería Civil y Mecánica...].

RETAMAL ARRIAZA, S. E. (2018). PROPUESTA DE PLAN DE MANTENIMIENTO PARA BOMBAS CENTRÍFUGAS UBICADAS EN ANGLO AMERICAN SUR PLANTA LAS TÓRTOLAS ESTACIÓN DE REBOMBEO.

VERA, L., JOHAN, D., \& ROJAS CEVALLOS, M. B. (2017). ÍNDICE

DE COMPETITIVIDAD EN EL ANÁLISIS COMPARATIVO DE LAS DIFERENTES EMPRESAS ATUNERAS DE MANTA, MONTECRISTI $Y$ JARAMIJÓ. 